\title{
The coordination value of monetary exchange: Experimental evidence
}

\author{
Gabriele Camera $^{\dagger}$ and Marco Casari $^{\ddagger}$
}

\section{Online Appendix}

\section{Baseline treatment: existence of cooperative equi- librium}

There are four identical players. In each period they are matched in pairs, with uniform probability of selection. In each pair, one agent is a seller (red) and the other is a buyer (blue). The roles are assigned through a coin flip.

Two outcomes are possible in a match: autarky $Y$ and cooperation $Z$. When $Z$ is the outcome, $u=20$ is the stage game payoff for a buyer and $-c=2$ for the seller. If $Y$ is the outcome, then both playrs earn $a=8$. Clearly, $\frac{u-c}{2}>a$. Period payoffs are geometrically discounted at rate $\beta=0.93$. Payoffs and continuation payoffs in the repeated game are given by expected lifetime utilities.

\subsection{Equilibrium payoffs}

Consider a social norm based on the grim trigger strategy. It has a rule for cooperation: a seller must always choose $Z$. It has also a rule for punishment: If a defection is observed, then a seller chooses $Y$ forever after. Suppose an equilibrium exists based on this social norm. The equilibrium payoff of the representative player is

$$
V:=\frac{u-c}{2(1-\beta)}
$$

\footnotetext{
$\dagger$ University of Basel and Economic Science Institute, Chapman University

${ }^{\ddagger}$ University of Bologna
} 
This is the present value of the stream of expected period payoffs.

To discuss existence of equilibrium we present individual optimality conditions in and out of equilibrium. In equilibrium, playing $Z$ is a best response for a seller if

$$
-c+\beta V \geq a+\beta v_{2}
$$

The left-hand-side denotes the payoff from cooperating in equilibrium. The right-hand-side has the payoff from defecting in equilibrium, where $v_{2}$ denotes the off-equilibrium continuation payoff in an economy where two agents have seen a defection and follow the rule of punishment of the social norm. Since $V>v_{2}$ for (2) to hold, we rewrite it as

$$
\beta \geq \beta_{L}:=\frac{a+c}{V-v_{2}}
$$

\subsection{Out of equilibrium payoffs}

Consider out of equilibrium actions when everyone follows the social norm. Clearly, out of equilibrium we have at least two defectors. Let $v_{4}$ denote the continuation payoff for any player in an economy with 4 defectors; we have

$$
v_{4}:=\frac{a}{1-\beta}
$$

and we call $v_{4}$ the autarky payoff.

Now consider the case where a defection has just taken place for the first time. At this point there are only two defectors. For concreteness, let agent $x$ observe a defection for the first time in period $t-1$. She believes that everyone has cooperated up to that point. Agent $x$ may be the one who defected, or her opponent, denoted $y$. Suppose that everyone behaves according to the social norm. Next period $t$ there will be two defectors (agents $x$ and $y$ ) and two cooperators (agents in the other match who observed nothing).

The continuation payoff for agent $x$ at the start of period $t$ is

$$
v_{2}=\frac{1}{3}\left(a+\beta v_{2}\right)+\frac{2}{3}\left[\frac{1}{2}\left(a+\beta \frac{v_{4}+v_{3}}{2}\right)+\frac{1}{2}\left(u+\beta \frac{v_{2}+v_{3}}{2}\right)\right] .
$$

With probability $\frac{1}{3}$ agent $x$ meets again agent $y$ (a defector), and with probability 
$\frac{2}{3}$ agent $x$ meets a cooperator.

- If $x$ meets $y$ once again, $a$ is the period payoff; hence, at the end of $t+1$ there will still be only two defectors and the discounted continuation payoff is $\beta v_{2}$.

- If $x$ does not meet $y$ once again, then he meets a cooperator.

- If $x$ is a seller (with probability $\frac{1}{2}$ ), then $x$ defects and earns $a$. The defection is seen by the opponent but the continuation payoffs depends also on what happens in the other match. The other pair is also composed of a defector (agent $y$ ) and a cooperator. If agent $y$ is a seller, then he defects. Hence, next period we have four defectors and $v_{4}$ is the continuation payoff. If, instead, agent $y$ is a buyer, then there is no defection in the other match and the following period we have three defectors and $v_{3}$ is the continuation payoff. Since $y$ is a seller with probability $\frac{1}{2}$, then this also the probability that a defection occurs in the other match.

- If $x$ is a buyer (with probability $\frac{1}{2}$ ), then he earns $u$ in the period. The continuation payoff depends on outcomes in the other match. Since $x$ does not defect, we cannot have more than three defectors next period. With probability $\frac{1}{2}$ there are three defectors; otherwise, there are two.

Rearrange (4) as

$$
v_{2}=\frac{2}{3(2-\beta)}\left(u+2 a+\beta \frac{1}{2} v_{4}+\beta v_{3}\right) .
$$

To calculate $v_{3}$ consider the out-of-equilibrium case when, at the beginning of some date, agent $x$ is one of three defectors. Suppose that everyone adopts the social norm. The payoff to agent $x$ is

$$
v_{3}=\frac{1}{3}\left[\frac{1}{2}\left(u+\beta v_{3}\right)+\frac{1}{2}\left(a+\beta v_{4}\right)\right]+\frac{2}{3}\left(a+\beta \frac{v_{4}+v_{3}}{2}\right),
$$


because with probability $\frac{1}{3}$ agent $x$ meets a cooperator, and with probability $\frac{2}{3}$ she meets a defector.

- If agent $x$ meets a cooperator, then her period earnings depends on whether she is a seller or a buyer. Her continuation payoff depends also on this because only if she sells will the economy move to the state with four defectors. Indeed, the other match has two defectors.

- If agent $x$ meets a defector, then she always earns $a$ but the continuation payoff depends on whether the cooperator in the other match is a buyer. If that's the case (with probability $1 / 2$ ), then the economy transitions to a state with four defectors. Otherwise, it will remain in a state with three defectors.

Rearranging (6) we have

$$
v_{3}=\frac{1}{3(2-\beta)}\left(u+5 a+3 \beta v_{4}\right) .
$$

Using the above in (4) we have

$$
v_{2}=\frac{2}{3(2-\beta)^{2}}\left\{(u+2 a)(2-\beta)+\beta\left[\frac{(2+\beta) a}{2(1-\beta)}+\frac{u+5 a}{3}\right]\right\} .
$$

We now determine a condition such that defecting in equilibrium is individually sub-optimal

Lemma 1. There exists a non-trivial interval $\left(\beta_{L}, 1\right)$ such that if $\beta \in\left(\beta_{L}, 1\right)$, then (2) holds.

Proof of Lemma 1. Rewrite (2) as $\frac{a+c}{v_{2}} \leq \beta\left(\frac{V}{v_{2}}-1\right)$. As $\beta \rightarrow 0$ we have $V \rightarrow \frac{u-c}{2}$ and $v_{2} \rightarrow \frac{u+2 a}{3}$. As $\beta \rightarrow 0$ then (2) is violated for any $a \geq 0$ and $c<0$. Notice that $\frac{\partial v_{2}}{\partial \beta}, \frac{\partial V}{\partial \beta}>0$. As $\beta \rightarrow 1$, we have $v_{2} \rightarrow \infty$ and $V \rightarrow \infty$. It should be clear that as $\beta \rightarrow 1$ then $\frac{a+c}{v_{2}} \rightarrow 0$. In addition, the RHS of the inequality converges to a positive quantity since, as $\beta \rightarrow 1$, then $\frac{V}{v_{2}} \rightarrow \frac{u-c}{2 a}>1$, given our initial assumption. We conclude that there exists a $\beta_{L}$ sufficiently close to one such that (2) holds for all $\beta \in\left(\beta_{L}, 1\right)$, with strict inequality. 


\subsection{Deviating out of equilibrium}

Here we find conditions under which it is optimal to follow the rule of punishment, out of equilibrium. We will consider a case when an agent who should defect may wish to cooperate, instead of defecting, to slow down the contagious punishment process.

Suppose on date $t$ agent $x$ observes a deviation for the first time; for convenience, let $y$ be agent's match (it does not matter which agent defects in the pair). Consider now the future date when agent $x$ is a seller for the first time after observing such defection. If this date is $t+1$, then there still are only two defectors in the economy (agents $x$ and $y$ ). This is the best-case scenario for someone who wishes to deviate from the punishment norm: if not punishing is suboptimal when there are only two defectors (including the deviant player), then it is also suboptimal when there are more than two defectors (the continuation payoff to the deviant can only be smaller).

Hence, consider the following one-time deviation out of equilibrium when there are two defectors, agents $x$ and $y$. Let agent $x$ be a seller who chooses $Z$ instead of $Y$ and reverts to following the social norm afterward. Choosing $Y$ is a best response if

$$
a+\beta\left(\frac{1}{3} v_{2}+\frac{2}{3} \frac{v_{3}+v_{4}}{2}\right) \geq-c+\beta\left(\frac{1}{3} v_{2}+\frac{2}{3} \frac{v_{2}+v_{3}}{2}\right) .
$$

- The LHS of (8) reports the payoff to $x$ when, as a seller, he follows the social norm, out of equilibrium. Agent $x$ defects and earns $a$ for the current period. The continuation payoff depends on whom she meets. With probability $\frac{1}{3}$ agent $x$ meets $y$ (who is also a defector), hence the continuation payoff is $v_{2}$ since the other match has two cooperators. If, instead, agent $x$ meets a cooperator (with probability $\frac{2}{3}$ ), then from next period the economy will have three defectors only if in the other match the defector $y$ is a buyer (with probability $\frac{1}{2}$ ).

- The RHS of (8) reports the payoff to $x$ when he chooses $Z$ today and $Y$ forever after. He earns $-c$ the current period. His continuation payoff 
depends on whom she meets. If she meets agent $y$ (who is also a defector), then next period there will be again two defectors ( $x$ and $y$ ). This occurs with probability $\frac{1}{3}$. If, instead, agent $x$ meets a cooperator, with probability $\frac{2}{3}$, next period the economy has 2 or 3 defectors depending on what happens in the other match. With probability $\frac{1}{2}$ there is no defection in the other match (agent $y$ is a buyer) and so next period there will be again only 2 defectors $(x$ and $y)$. Otherwise, there will be $3(x, y$ and the additional agent infected by $y)$.

Inequality (8) can be rearranged as

$$
a+c \geq \frac{\beta}{3}\left(v_{2}-v_{4}\right)
$$

Since $v_{2}>v_{4}$ for (9) to hold, we rewrite it as

$$
\beta \leq \beta_{H}:=\frac{3(a+c)}{v_{2}-v_{4}} .
$$

Proposition 1. For the parameterization $u=20, a=8$ and $-c=2$ the grim trigger strategy is an equilibrium for all $\beta \geq 0.808$.

Proof: Inserting $u=20,-c=2$ and $a=8$ we numerically find $\beta_{L}=0.808$ and $\beta_{H}=1.2$.

\section{The long-run distribution of tickets}

Conjecture that everyone adopts the fiat monetary exchange strategy. The distribution of tickets in the economy varies from period to period, depending on the distribution at the start of a period and the random matching. Given a constant supply of four tickets, there can be three possible distributions (states) at the start of a period denoted

$$
d_{1}=(2,2,0,0), d_{2}=(2,1,1,0), d_{3}=(1,1,1,1) .
$$

Let $p_{j}(t)$ denote the probability that state $d_{j}$ with $j=1,2,3$ is realized in period t. Since we want to study long-run outcomes, consider long-run probabilities, 
i.e., $p_{j}(t)=p_{j}(t+1)=p_{j}$.

Suppose the distribution is $d_{1}=(2,2,0,0)$. Three cases are possible, denoted $d_{1 a}, d_{1 b}, d_{1 c}$, because buyer $(B)$ and seller $(S)$ roles are randomly assigned:

$$
\begin{array}{cccccl} 
& B & B & S & S & \\
d_{1 a}: & 2 & 2 & 0 & 0 & \text { (exchange always feasible) } \\
d_{1 b}: & 2 & 0 & 2 & 0 & \text { (exchange can be unfeasible) } \\
d_{1 c}: & 0 & 0 & 2 & 2 & \text { (exchange always unfeasible) }
\end{array}
$$

Because buyer and seller roles are equally probable for each player, each of these three distributions arises with probability $1 / 3$. Given $d_{1 a}$, ticket exchange is always feasible. Given $d_{1 c}$ ticket exchange is never feasible. Given $d_{1 b}$, there are two cases to consider, each of which is equally likely, depending on random matching results. Denote $(B=x, S=y)$ the ticket holdings $x, y$ in a match. We have

$$
\begin{array}{ll}
\{(B=2, S=2),(B=0, S=0)\} & (\text { exchange always unfeasible) } \\
\{(B=2, S=0),(B=0, S=2)\} & \text { (exchange feasible in } 1 \text { match) }
\end{array}
$$

Let $p_{j, k}$ denote the probability of reaching state $d_{j}$ with $j=1,2,3$ conditional on being in state $k=1,2,3$. The discussion above implies

$$
\left(p_{1,1}, p_{2,1}, p_{3,1}\right)=\left(\frac{1}{2}, \frac{1}{6}, \frac{1}{3}\right) .
$$

Suppose the distribution is $d_{2}=(2,1,1,0)$. Three possible cases arise each with probability $1 / 3$ :

$$
\begin{array}{lccccl} 
& B & B & S & S & \\
d_{2 a}: & 2 & 1 & 1 & 0 & \text { (exchange always feasible) } \\
d_{2 b}: & 2 & 0 & 1 & 1 & \text { (exchange feasible in } 1 \text { match) } \\
d_{2 c}: & 1 & 0 & 2 & 1 & \text { (exchange can be unfeasible) }
\end{array}
$$

Given $d_{2 a}$, ticket exchange is always feasible and we go back to the same distribution $d_{2}$. Given $d_{2 b}$, ticket exchange is unfeasible in one match, and we also go back to the same distribution of tickets $d_{2}$. Given $d_{2 c}$, two equally probable 
cases may arise:

$$
\begin{array}{ll}
\{(B=1, S=2),(B=0, S=1)\} & \text { (exchange always unfeasible) } \\
\{(B=1, S=1),(B=0, S=2)\} & \text { (exchange feasible in } 1 \text { match). }
\end{array}
$$

From the discussion above, we have

$$
\left(p_{1,2}, p_{2,2}, p_{3,2}\right)=\left(\frac{1}{6}, \frac{5}{6}, 0\right)
$$

Suppose the distribution is $d_{3}=(1,1,1,1)$. Ticket exchange is feasible in every match, so

$$
\left(p_{1,3}, p_{2,3}, p_{3,3}\right)=(1,0,0)
$$

We can now calculate the long-run distribution of tickets, i.e., the unconditional probability of being in state $d_{j}$ with $j=1,2,3$. This must satisfy $p_{j}(t+1)=p_{j}(t)=p_{j}$ for all $j$ and all $t$, hence $\left\{p_{j}\right\}$ must solve $p_{3}=1-p_{1}-p_{2}$ and $p_{j}=\sum_{k=1}^{3} p_{k} p_{j, k}$ for each $j=1,2,3$. Since one equation is redundant, we must have

$$
\begin{aligned}
& p_{1}=p_{1} p_{1,1}+p_{2} p_{1,2}+\left(1-p_{1}-p_{2}\right) p_{1,3} \\
& p_{2}=p_{1} p_{2,1}+p_{2} p_{2,2} .
\end{aligned}
$$

Substituting for $p_{j, k}$ from above, we have

$$
p_{1}=p_{2}=p=3 / 7 \text { and } p_{3}=1 / 7 \text {. }
$$

To calculate the unconditional probability distribution of tickets in the economy we proceed as follows. Let $m_{i}$ denote the probability that in the long-run a player randomly selected from the population has $i=0,1,2$ tickets. We have:

$$
\begin{array}{llll}
m_{0}:=p_{1} \frac{1}{2}+p_{2} \frac{1}{4} & =9 / 28 & \simeq 0.321 \\
m_{1}:=p_{2} \frac{1}{2}+p_{3} & =1-9 / 14 & \simeq 0.357 \\
m_{2}:=1-m_{0}-m_{1} & =9 / 28 & \simeq 0.321 .
\end{array}
$$

To explain (10), consider the equation for $m_{0}$. Holdings of zero tickets are observed only in states $d_{1}$ and $d_{2}$. Each of these states occurs with (unconditional) probability $p$. In state $d_{1}$ only two players out of four have 0 tickets. Hence, the probability of observing an agent with zero tickets is 0.50 . In state $d_{2}$, only 
one agent out of four has 0 ticket holdings. The probability of observing 0 ticket holdings is thus 0.25 . The second equation in (10) can be similarly explained. In the experiment we have $\left(m_{0}, m_{1}, m_{2}\right)=(0.315,0.371,0.315)$ as reported in Table 7 .

The long-run fraction of matches in which ticket exchange is unfeasible can now be calculated. Ticket exchange may be unfeasible only in states $d_{1}$ and $d_{2}$. Consider state $d_{1}$. Ticket exchange (i) is always unfeasible if $d_{1 c}$ is the distribution, (ii) is never unfeasible if $d_{1 a}$ is the distribution, while (iii) if $d_{1 b}$ is the distribution, then in one subcase ticket exchange is always unfeasible, and in the other it is unfeasible only in one match (each subcase is equally likely). Recall that the substates $d_{1 i}, i=a, b, c$, are equally probable. Consequently, in state $d_{1}$ the anticipated proportion of matches in which exchange is unfeasible is denoted $\phi_{1}$ where

$$
\phi_{1}:=\operatorname{Pr}\left[d_{1 c}\right]+\operatorname{Pr}\left[d_{1 b}\right] \times\left(\frac{1}{2}+\frac{1}{2} \times \frac{1}{2}\right) \simeq 58.33 \% .
$$

A similar approach applied to state $d_{2}$ gives us that the anticipated proportion of matches in which exchange is unfeasible is

$$
\phi_{2}:=\operatorname{Pr}\left[d_{2 b}\right] \frac{1}{2}+\operatorname{Pr}\left[d_{2 c}\right] \times\left(\frac{1}{2}+\frac{1}{2} \times \frac{1}{2}\right) \simeq 41.66 \% .
$$

Considering that states $d_{1}$ and $d_{2}$ occur with probability $p_{1}=p_{2}=p$, the expected fraction of matches in which ticket exchange is unfeasible, in the long, run is given by:

$$
p_{1} \phi_{1}+p_{2} \phi_{2}
$$

which amounts to $p$ once we substitute for $\phi_{1}$ and $\phi_{2}$.

\section{Long-run monetary equilibrium in the Tickets treat- ment}

Consider the following history-independent strategy: a seller who has less than 2 tickets chooses $Z$ conditional on receiving one ticket; a buyer offers one unit of money conditional on the seller choosing $Z$. In all other circumstances, a seller 
chooses $Y$ and the buyer does not offer any ticket. We conjecture existence of a long-run equilibrium in which this strategy is adopted by everybody.

To focus on long-run equilibrium we must calculate the long-run value of tickets as an unconditional expectation, considering the long-run distribution of tickets. Let $m_{i}$ denote the long-run probability that a seller is matched to a buyer who has $i=0,1,2$ tickets. By virtue of our conjectured strategy (in equilibrium sellers choose $Z$ in exchange for a ticket, only if they have less than 2 tickets) the values $m_{j}$ are given by (10). Denote by $V_{i}$ the long-run expected value of holding $i$ tickets. Recursively, we have

$$
\begin{aligned}
& V_{2}=a+\beta V_{2}+\frac{1-m_{2}}{2}\left[u-a-\beta\left(V_{2}-V_{1}\right)\right] \\
& V_{1}=a+\beta V_{1}+\frac{1-m_{0}}{2}\left[-c-a+\beta\left(V_{2}-V_{1}\right)\right]+\frac{1-m_{2}}{2}\left[u-a-\beta\left(V_{1}-V_{0}\right)\right] \\
& V_{0}=a+\beta V_{0}+\frac{1-m_{0}}{2}\left[-c-a+\beta\left(V_{1}-V_{0}\right)\right]
\end{aligned}
$$

Consider $V_{1}$. Each player is matched in each period. If he is a seller (with probability $\frac{1}{2}$ ), then the opponent is a buyer, and vice-versa. The player can always assure himself the autarky payoff $a+\beta V_{1}$. In addition, the agent can earn some surplus over autarky. If the agent is a seller, then he cooperates in exchange for one ticket only if he meets someone who has tickets, with probability $1-m_{0}$. Choosing $Z$ gives the seller utility $-c$ plus continuation payoff $\beta V_{2}$ because a ticket is received. The opportunity cost is the autarky payoff $a+\beta V_{1}$. Hence, the surplus as a seller is $-c-a+\beta\left(V_{2}-V_{1}\right)$. With probability $\frac{1}{2}$ the player; in equilibrium he earns surplus $u-a-\beta\left(V_{1}-V_{0}\right)$ only if he meets someone (a seller) with less than two tickets, with probability $1-m_{2}$. The payoffs $V_{0}$ and $V_{2}$ are similarly explained.

The monetary exchange strategy is an equilibrium if surpluses of buyer and seller are non-negative, i.e., if spending a unit of money is individually optimal for buyers, and cooperating in exchange for money is individually optimal for sellers. We have:

$$
\beta\left(V_{i+1}-V_{i}\right) \leq u-a \text { and } a+c \leq \beta\left(V_{i+1}-V_{i}\right) \text { for } i=0,1
$$

To prove that the conjectured monetary equilibrium exists, we need to find 
conditions, in terms of the parameters of the model, sufficient to satisfy the four inequalities in (12).

Start by noticing that we must only check two inequalities in (12), which are

$$
\beta\left(V_{1}-V_{0}\right) \leq u-a \text { and } a+c \leq \beta\left(V_{2}-V_{1}\right)
$$

To see this note that if the inequalities in (13) hold, then the remaining two inequalities in (12) hold because $V_{1}-V_{0} \geq V_{2}-V_{1}$. To prove it, let (13) be true. From (11) we get

$$
\begin{aligned}
& V_{2}-V_{1}=\frac{\phi}{2}\left[\left(1-m_{0}\right)(a+c)+\left(1-m_{2}\right) \beta\left(V_{1}-V_{0}\right)\right] \\
& V_{1}-V_{0}=\frac{\phi}{2}\left[\left(1-m_{0}\right) \beta\left(V_{2}-V_{1}\right)+\left(1-m_{2}\right)(u-a)\right],
\end{aligned}
$$

where we define

$$
\phi:=\frac{2}{2-\beta\left(m_{0}+m_{2}\right)} \in(1,2) .
$$

Hence, if the inequalities in (13) hold then from (14) we have $V_{1}-V_{0} \geq V_{2}-V_{1}$.

Now note that the two inequalities in (13) can be rewritten as

$$
a \leq \min \left(u-\beta\left(V_{1}-V_{0}\right),-c+\beta\left(V_{2}-V_{1}\right)\right) .
$$

Hence, in general there is only one condition to check. We claim that $a \leq-c+$ $\beta\left(V_{2}-V_{1}\right)$ is the binding condition. We prove it by contradiction. Suppose that we have a monetary equilibrium where $a<-c+\beta\left(V_{2}-V_{1}\right)$ but $a=u-\beta\left(V_{1}-V_{0}\right)$, i.e., the other inequality is the one that binds.

If $0=u-a-\beta\left(V_{1}-V_{0}\right)$, then (11) implies $V_{1} \leq V_{0}$ (because in monetary equilibrium $\left.V_{1}-V_{0} \geq V_{2}-V_{1}\right)$. But this contradicts the conjecture $a=u-$ $\beta\left(V_{1}-V_{0}\right)$ because $a<u$. Hence, we can only have $a=-c+\beta\left(V_{2}-V_{1}\right)$ and $a<u-\beta\left(V_{1}-V_{0}\right)$. To sum up, to find parameters for the existence of a monetary equilibrium we must check

$$
0 \leq-a-c+\beta\left(V_{2}-V_{1}\right) .
$$

This condition says that a seller with one ticket chooses to cooperate in exchange 
for another ticket. Rewrite $V_{2}-V_{1}$ as

$$
V_{2}-V_{1}=\frac{\phi\left[\frac{1}{2}\left(1-m_{0}\right)(a+c)+\phi\left(\frac{1}{2}\right)^{2}\left(1-m_{2}\right)^{2}(u-a) \beta\right]}{1-\left(\frac{\phi}{2}\right)^{2}\left(1-m_{2}\right)\left(1-m_{0}\right) \beta^{2}} .
$$

A monetary equilibrium exists both for the case of the theoretical distribution in (10) as well as for the case of the empirical distribution. The right hand side in $(15)$ is 0.448 , for the theoretical distribution, and 0.446 for the empirical distribution.

To prove that in equilibrium the ticket holding constraint is not binding, consider a case when, out of equilibrium an agent has three instead of two tickets. Hence,

$$
V_{3}=a+\beta V_{3}+\frac{1-m_{2}}{2}\left[u-a-\beta\left(V_{3}-V_{2}\right)\right],
$$

because this agent will buy if he has a chance, but will not cooperate in exchange of a ticket (as a seller). Proceeding as above we have

$$
V_{3}-V_{2}=\frac{\frac{1}{2} m_{0} \beta\left(V_{2}-V_{1}\right)}{1-\beta+\frac{1}{2} m_{0} \beta} .
$$

A seller with 2 tickets does not cooperate if $-c+\beta V_{3}<a+\beta V_{2}$, rewritten as

$$
\frac{\frac{1}{2} m_{0} \beta^{2}\left(V_{2}-V_{1}\right)}{1-\beta+\frac{1}{2} m_{0} \beta}<a+c .
$$

Using $V_{2}-V_{1}$ from (16) one can verify that (17) holds for the parameters in the experiment. 


\section{Appendix - Instructions for Baseline Treatment}

This is an experiment in decision-making. The University of Iowa has provided funds for this research. You can earn money based on how well you follow the instructions and on the decisions you make in the experiment. Please turn off your cell-phones, do not talk to others and do not look at their screens. These instructions are a detailed description of the procedures we will follow.

\section{How do you earn money?}

You will earn points that will be converted into dollars. You will receive 2.5 cents $(\$ .025)$ for every point you earn. All earnings will be paid to you in cash at the end of the experiment.

The experiment is composed of many periods. In each period you will be in a pair with another person selected at random, called your "match." In every pair, one participant will be red and the other blue:

- If you are red, then you can choose to execute either outcome $\mathbf{Y}$ or $\mathbf{Z}$ :

- By choosing to execute outcome $\mathbf{Y}$, you earn $\boldsymbol{8}$ points and your blue match earns $\boldsymbol{8}$ points.

- By choosing to execute outcome $\mathbf{Z}$, you earn 2 points and your blue match earns 20 points.

- If you are blue, then you simply wait for red to make a choice.

You can expect to be red $50 \%$ of the periods and blue the other $50 \%$.

Who will be your match in the pair?

There are twenty participants. Each participant will be assigned to a set composed of four persons:

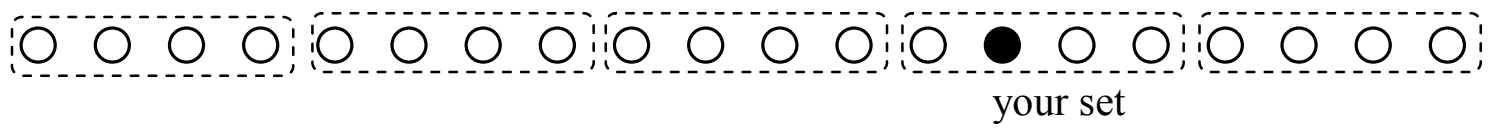

There are five sets. Your match is a person chosen at random from your set. The computer program selects with equal probability one of the three other persons in your set. So, there is one chance out of three that your match is any of the other three persons in your set.

Although there is a possibility that you interact with the same participant more than once, you will not know if it happens. Your match will be unknown to you because you will not see his or her experimental ID number.

In every period, after your match is selected, the computer randomly selects your color. In every period you have a $50 \%$ chance to be red and $50 \%$ chance to be blue. Your randomly selected match is always of a different color than yours. Hence in every set, two persons are red and the other two are blue. Since the color assignment is random, you may or may not switch color from period to period. 


\section{How many periods will the experiment last?}

The experiment consists of five cycles. Each cycle involves many periods |||||| :

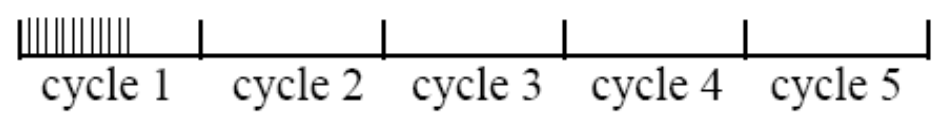

The number of periods in a cycle is random and so it is unknown to us. At the end of each period, the computer program randomly selects an integer number between 1 and 100. Each number is equally likely to be selected. This random number is the same for everyone in the room.

The cycle ends only if the random number selected is greater than 93 . This means that:

- We never know for sure which period will be the last in a cycle.

- After each period there is a $93 \%$ chance that the cycle continues and a $7 \%$ chance that the cycle ends.

- Some cycles may be long and others may be short, but we cannot know this in advance.

The computer will select the random number in the same way a ball is drawn from a container of onehundred balls, numbered 1 to 100. After each draw the ball is placed back into the container. Hence, the chance that a cycle will end, say, after period 25 , is $7 \%$, which is exactly the same as the chance that the cycle will end after period 1.

When a cycle ends, all twenty participants are divided into new sets in such a way that you will face different participants. You will never interact with the same participants in future cycles.

\section{What exactly will you do in each period?}

Each period has the following timeline:

1. You are randomly paired to a participant from your set.

2. You are randomly assigned a color (red or blue).

3. You may be called to make a choice (see below).

4. You and your match see the outcome of your choices.

5. The cycle may continue or may end.

In a moment we will explain the choices you may make in each period. The choices depend on your color, red or blue. Remember that if you are red, then your match is blue (and vice versa). 
- If you are red, then you can select one of the following options (Figure A):

- Execute Y: you and your match earn $\boldsymbol{8}$ points each.

- Execute Z: you earn 2 points and your blue match earns 20 points.

- If you are blue, then you simply wait (Figure B)

Figure A: Choice screen for red

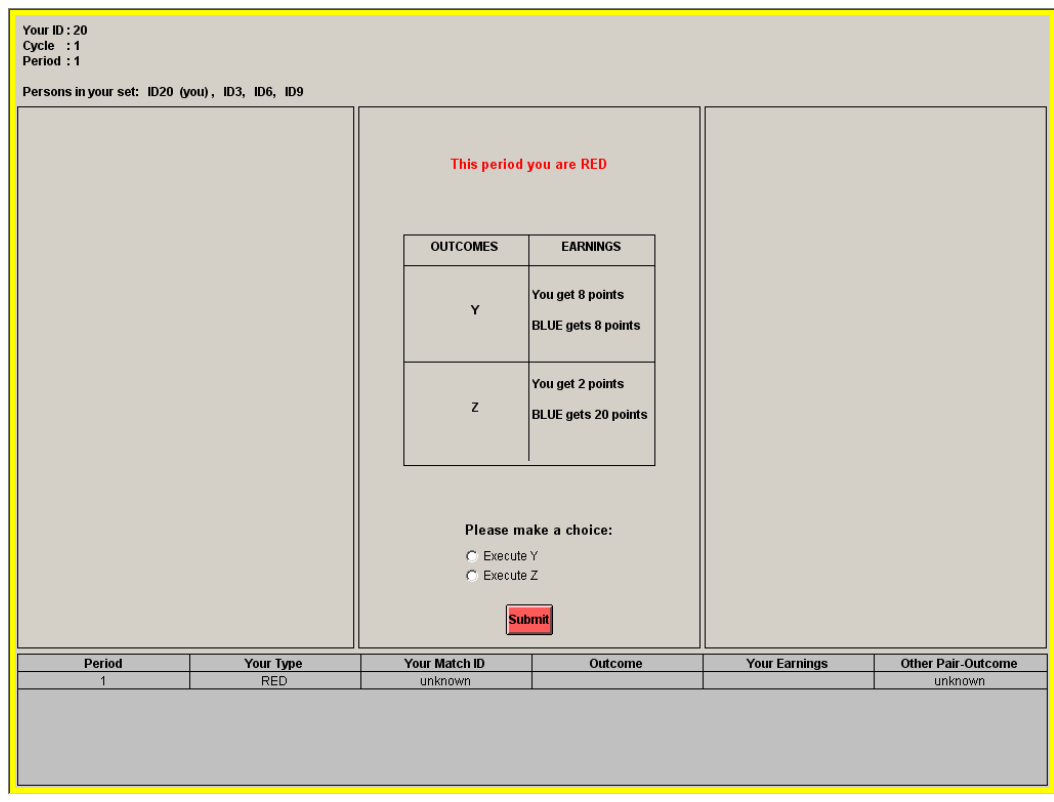

Figure B: Choice screen for blue

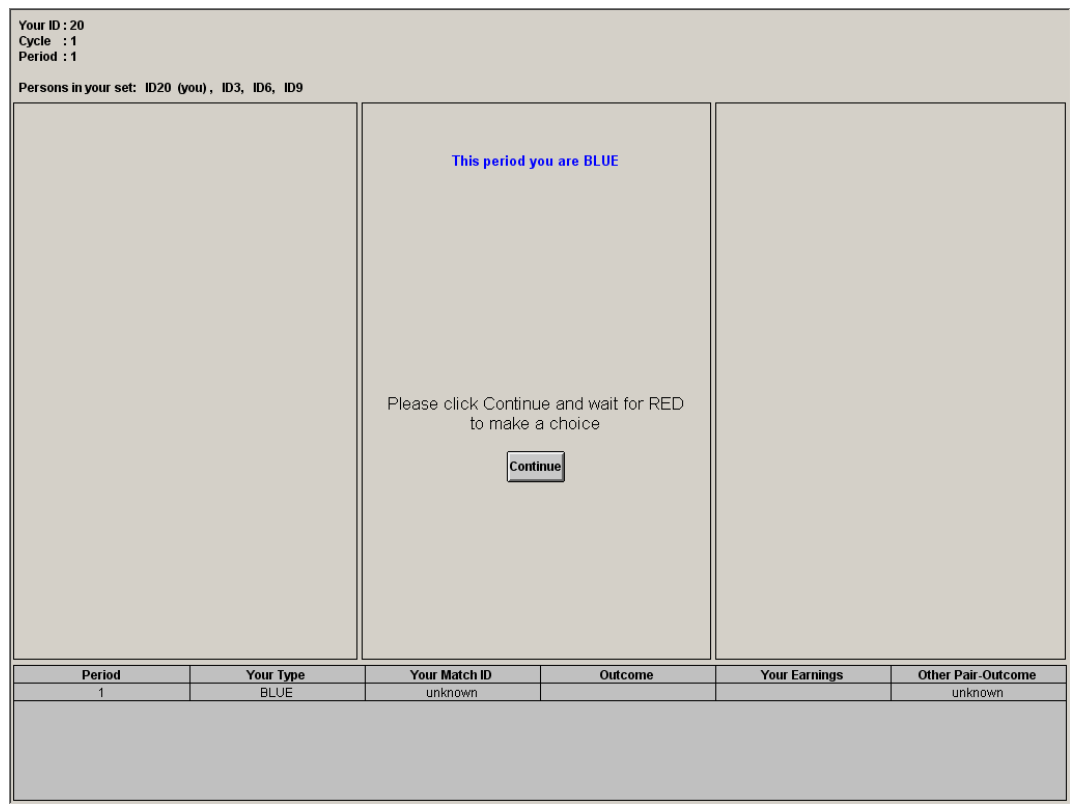


To make your choice, click the button next to the option you wish to select. You may change your mind at any time prior to clicking the "Submit" button. You are free to make any choice you like in each period.

Before making your choices, you can also review outcomes in previous periods of the cycle by looking at the "Summary of Results" table at the bottom of the screen. It shows your past colors and outcomes.

After all participants in your set have made their choice, the results for the period will appear on your screen:

The results screen (Figure C) will display your earnings in points for the period. You can see if the outcome was $\mathbf{Y}$ or $\mathbf{Z}$. The table in the lower part of the screen shows a "Summary of Results" for previous periods. Each line includes: period number, your color for the period, and the outcome $\mathbf{Y}$ or Z. The column "Your Earnings" displays the points you have earned. Recall that your match this period may be a different person than your match in the previous period. Please write the results on your record sheet under the appropriate headings.

\section{Figure C: Screen for the results of the period:}

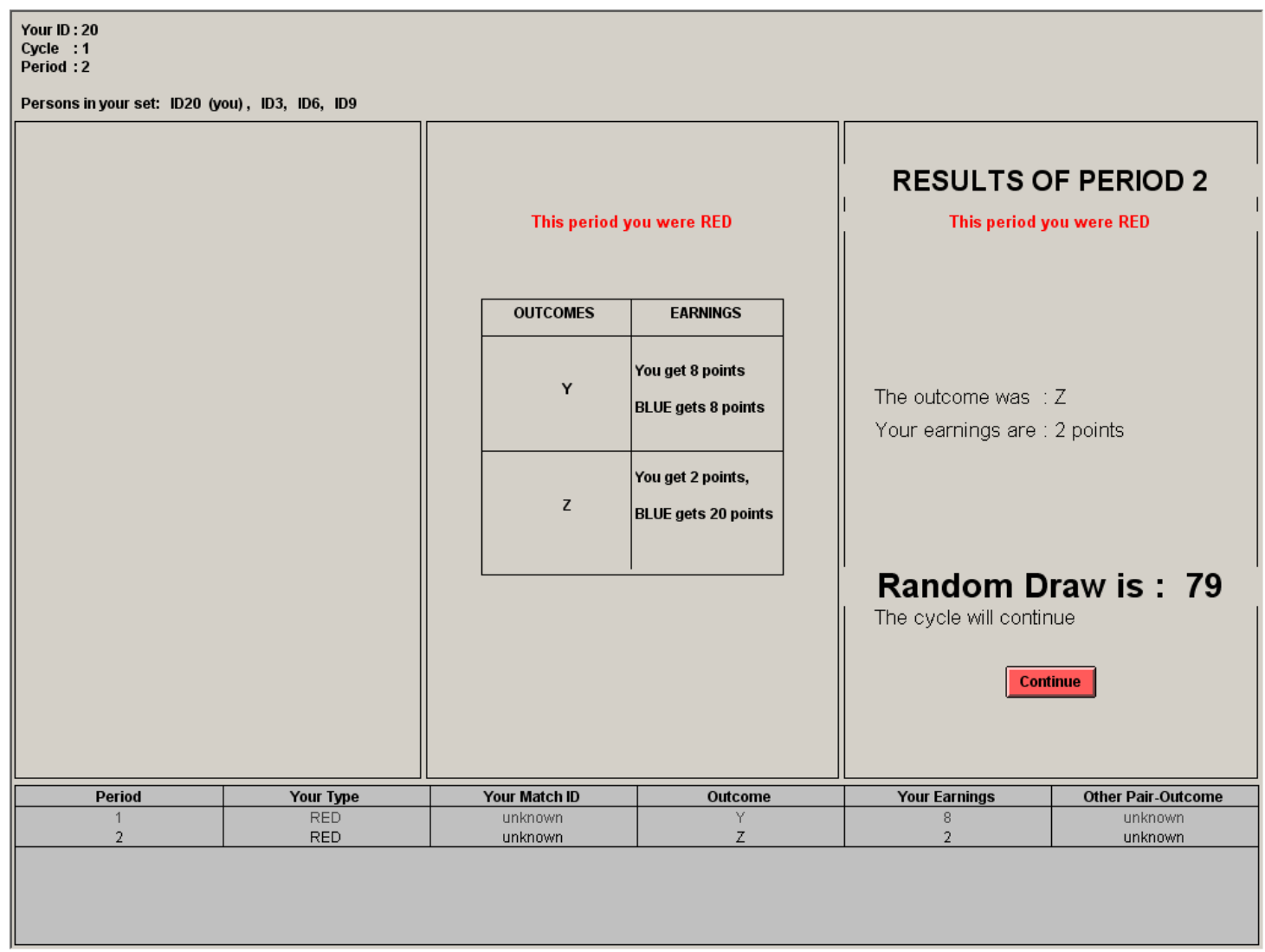




\section{Reminder on the duration of the experiment}

There will be five cycles of unknown duration. The duration of each cycle will be random. At the end of each period, the computer program will randomly select an integer number between 1 and 100, and show it on your screen (Figure C).

- If this random number is $1,2, \ldots$, or 93 , then the cycle will continue.

- If this random number is $94,95, \ldots$, or 100 , then the cycle will end.

Therefore, after any period there is always a 93\% chance that the cycle will continue. This implies that, no matter what period you have reached, the expected number of additional periods is about 13. The number of past periods does not influence the chance that a cycle will end because the random procedure is exactly the same in every period.

When a cycle ends, you will be notified in a new screen. The rules in each cycle are the same but you interact with different persons in each cycle. After each cycle, new sets of persons will be formed. You will never interact with another participant for more than one cycle.

\section{Final Comments}

- Do not talk to others and do not look at their screens.

- In every period you have a 50\% chance to be red and $50 \%$ chance to be blue.

- If you are red, then you can choose to execute either outcome $\mathbf{Y}$ or $\mathbf{Z}$. If you are blue, then you simply wait. Earned points will be redeemed for dollars.

- Your match is a random person in your set. You have one chance out of three of being matched with the same person in two consecutive periods.

- Independently of the period reached, there is a 93\% chance of an additional period in the cycle, and a $7 \%$ chance that the cycle ends.

- The rules are the same in all five cycles. After a cycle, you will never interact with the same participants.

\section{Questions?}

Now is time for questions. Do you have any questions before we begin the experiment? 


\section{QUIZ}

1. The total number of cycles is

2. You are in period 1 of a cycle. What is the probability that the cycle will continue? How many additional periods do we expect?

3. What if you are in period 20? Probability Expected additional periods

4. The number of participants in the experiment (total in the room) is

5. In a given cycle, how many participants are in your set?

6. In each period how many participants do you interact with?

7. Will you ever see the ID of your match?

8. Can you see how many times your match chose $\mathrm{Y}$ or $\mathrm{Z}$ in the past? _No_If all pay _If I pay (circle one)

9. Will you know at the end of the period the outcome in the other pair from your set?

10. If IDs 5, $10 \& 16$ are in your set this cycle, is there any chance that ID 5, 10 or 16 will be your match in future cycles?

11. You are BLUE and your RED match executes $\mathbf{Y}$; how many points do you earn, respectively?

12. Suppose the experiment lasts 70 periods, you are RED half of the periods, BLUE half of the periods, and everybody always chooses $\mathbf{Y}$. How many dollars will you earn?

13. RED chooses $\mathbf{Z}$; how many points do RED and BLUE earn?

14. Suppose the experiment lasts 70 periods, you are RED half of the periods, BLUE half of the periods, and everybody always chooses Z Z. How many dollars will you earn? 


\begin{tabular}{|c|c|c|c|c|c|c|}
\hline Cycle & Period & $\begin{array}{l}\text { Your } \\
\text { color }\end{array}$ & $\begin{array}{c}\text { Your Choice } \\
\text { Blue: -- } \\
\text { Red: Y, Z }\end{array}$ & Outcome & $\begin{array}{c}\text { Your period } \\
\text { earnings }\end{array}$ & $\begin{array}{c}\text { Cumulative } \\
\text { earnings }\end{array}$ \\
\hline & & & & & & \\
\hline & & & & & & \\
\hline & & & & & & \\
\hline & & & & & & \\
\hline & & & & & & \\
\hline & & & & & & \\
\hline & & & & & & \\
\hline & & & & & & \\
\hline & & & & & & \\
\hline & & & & & & \\
\hline & & & & & & \\
\hline & & & & & & \\
\hline & & & & & & \\
\hline & & & & & & \\
\hline & & & & & & \\
\hline & & & & & & \\
\hline & & & & & & \\
\hline & & & & & & \\
\hline & & & & & & \\
\hline & & & & & & \\
\hline & & & & & & \\
\hline & & & & & & \\
\hline & & & & & & \\
\hline & & & & & & \\
\hline & & & & & & \\
\hline & & & & & & \\
\hline & & & & & & \\
\hline & & & & & & \\
\hline & & & & & & \\
\hline & & & & & & \\
\hline & & & & & & \\
\hline & & & & & & \\
\hline & & & & & & \\
\hline & & & & & & \\
\hline
\end{tabular}




\section{Appendix - Instructions for Tickets treatment}

This is an experiment in decision-making. The University of Iowa has provided funds for this research. You can earn money based on how well you follow the instructions and on the decisions you make in the experiment. Please turn off your cell-phones, do not talk to others and do not look at their screens. These instructions are a detailed description of the procedures we will follow.

\section{How do you earn money?}

You will earn points that will be converted into dollars. You will receive 2.5 cents $(\$ .025)$ for every point you earn. All earnings will be paid to you in cash at the end of the experiment.

The experiment is composed of many periods. In each period you will be in a pair with another person selected at random, called your "match." In every pair, one participant will be red and the other blue:

- If you are red, then you can choose to execute either outcome $\mathbf{Y}$ or $\mathbf{Z}$ :

- By choosing to execute outcome $\mathbf{Y}$, you earn $\boldsymbol{8}$ points and your blue match earns $\boldsymbol{8}$ points.

- By choosing to execute outcome $\mathbf{Z}$, you earn 2 points and your blue match earns 20 points.

- If you are blue, then you may choose whether to give a "ticket" to your red match, as discussed below.

You can expect to be red $50 \%$ of the periods and blue the other $50 \%$.

Who will be your match in the pair?

There are twenty participants. Each participant will be assigned to a set composed of four persons:

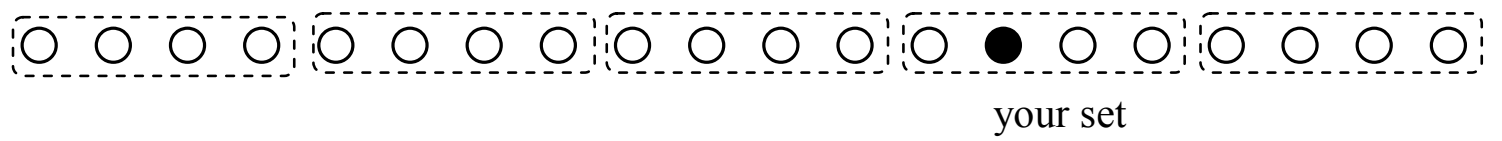

There are five sets. Your match is a person chosen at random from your set. The computer program selects with equal probability one of the three other persons in your set. So, there is one chance out of three that your match is any of the other three persons in your set.

Although there is a possibility that you interact with the same participant more than once, you will not know if it happens. Your match will be unknown to you because you will not see his or her experimental ID number.

In every period, after your match is selected, the computer randomly selects your color. In every period you have a $50 \%$ chance to be red and $50 \%$ chance to be blue. Your randomly selected match is always of a different color than yours. Hence in every set, two persons are red and the other two are blue. Since the color assignment is random, you may or may not switch color from period to period. 


\section{How many periods will the experiment last?}

The experiment consists of five cycles. Each cycle involves many periods $\|||||$ :

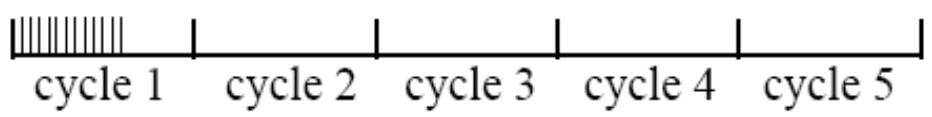

The number of periods in a cycle is random and so it is unknown to us. At the end of each period, the computer program randomly selects an integer number between 1 and 100. Each number is equally likely to be selected. This random number is the same for everyone in the room.

The cycle ends only if the random number selected is greater than 93. This means that:

- We never know for sure which period will be the last in a cycle.

- After each period there is a $93 \%$ chance that the cycle continues and a $7 \%$ chance that the cycle ends.

- Some cycles may be long and others may be short, but we cannot know this in advance.

The computer will select the random number in the same way a ball is drawn from a container of onehundred balls, numbered 1 to 100. After each draw the ball is placed back into the container. Hence, the chance that a cycle will end, say, after period 25 , is $7 \%$, which is exactly the same as the chance that the cycle will end after period 1.

When a cycle ends, all twenty participants are divided into new sets in such a way that you will face different participants. You will never interact with the same participants in future cycles.

At the beginning of each cycle every participant who is blue will receive two tickets. Participants can exchange tickets in any cycle. When a cycle is complete, tickets cannot be redeemed for dollars.

\section{What exactly do you need to do in each period?}

Each period has the following timeline:

1. You are randomly paired to a participant from your set.

2. You are randomly assigned a color (red or blue).

3. You and your match make simultaneous choices (see below).

4. You and your match see the outcome of your choices.

5. The cycle may continue or may end.

In a moment we will explain the choices you may make in each period. The choices depend on your color, red or blue, and on the number of tickets you have. Remember that if you are red, then your match is blue (and vice versa), and that you and your match make simultaneous choices. 
- If you are red, then you can select one of the following options (Figures A and B):

- Execute Y: you and your match earn 8 points each.

- Execute Z: you earn 2 points and your blue match earns 20 points.

- Execute $\mathbf{Z}$ only if blue gives me a ticket: If your blue match transfers a ticket to you, then the outcome is $\mathbf{Z}$. Otherwise, the outcome is $\mathbf{Y}$.

Note: You can hold at most two tickets. So, if you have two tickets, then you may not ask for another one; hence the last choice will not appear on your screen.

- If you are blue and do not have a ticket, you simply wait. Otherwise, you may select one of the following options:

- Keep your ticket(s)

- Give a ticket to red

- Give a ticket to red only if red executes $\mathrm{Z}$.

\section{Figure A: Choice screen for red}

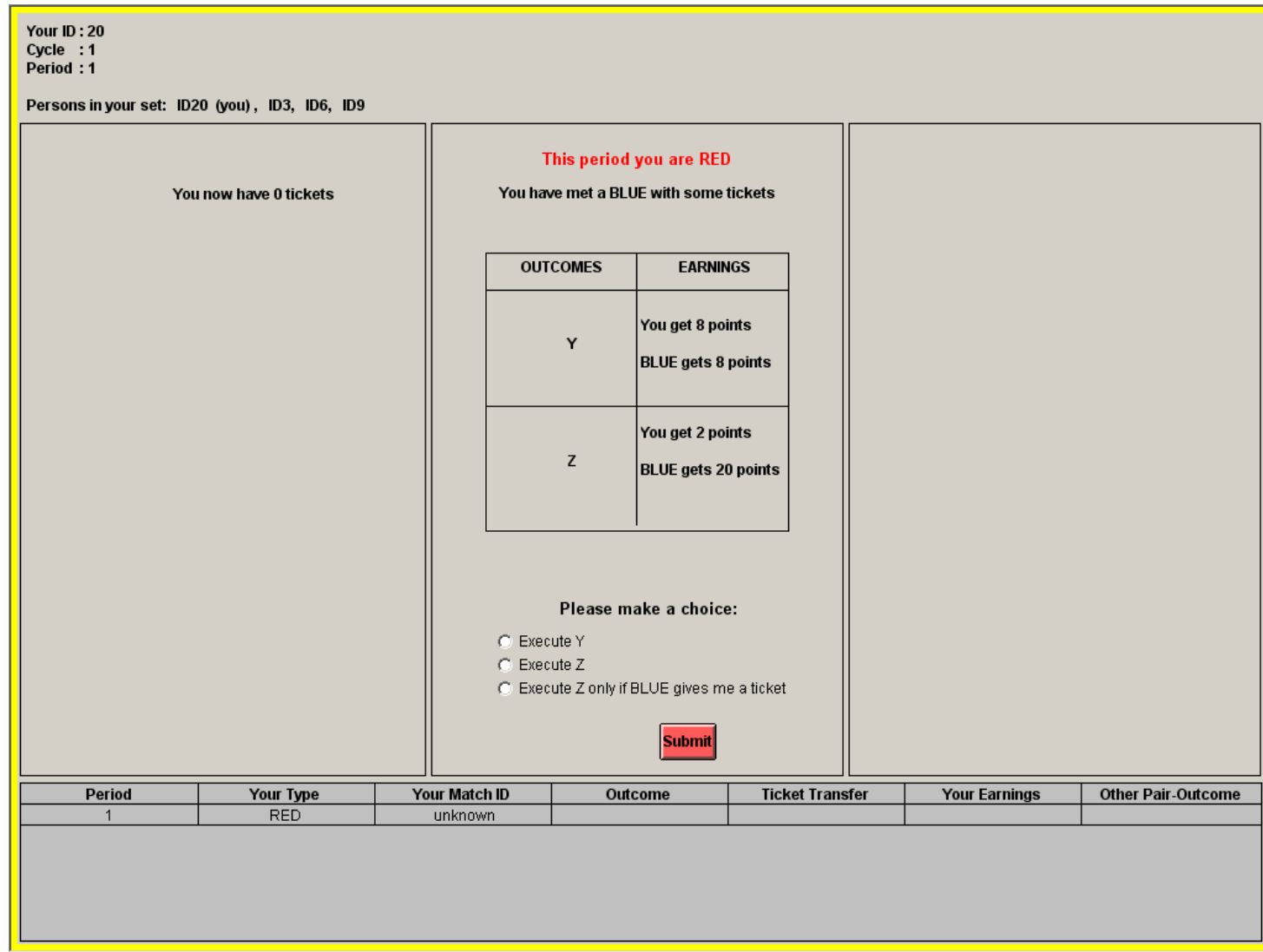




\section{Figure B: Choice screen for blue}

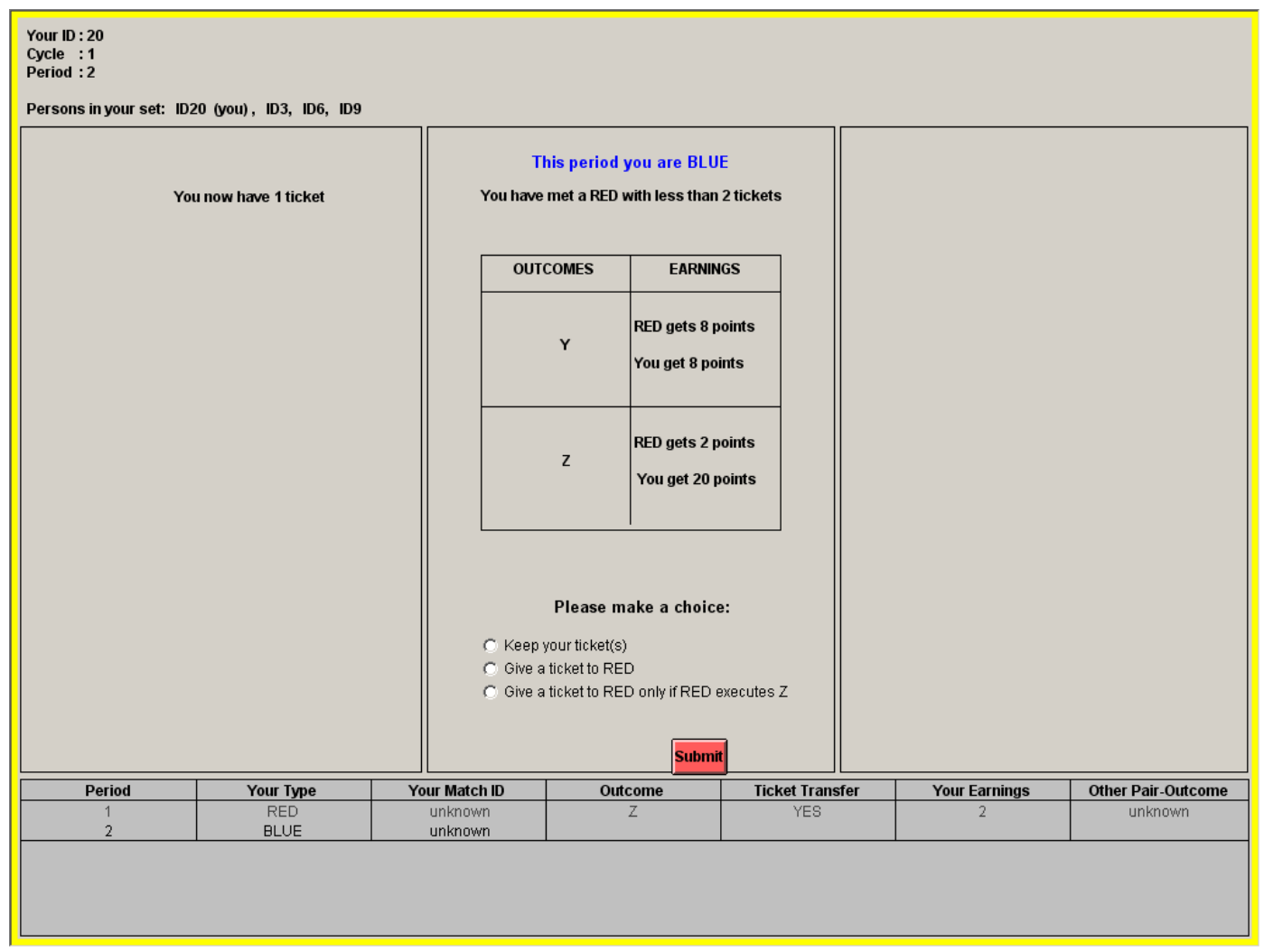

To make your choice, click the button next to the option you wish to select. You may change your mind at any time prior to clicking the "Submit" button. You are free to make any choice you like in each period.

Before making your choices, you can also review outcomes in previous periods of the cycle by scrolling down the "Summary of Results" table. The table shows your past colors and outcomes.

After all participants in your set have made their choice, the results for the period will appear on your screen:

The results screen (Figure C) will display your earnings in points for the period. You can see if the outcome was $\mathbf{Y}$ or $\mathbf{Z}$ and if any ticket was transferred. The table in the lower part of the screen shows a "Summary of Results" for previous periods. Each line includes: period number, your color for the period, and the outcome $\mathbf{Y}$ or $\mathbf{Z}$. If there was a ticket transfer, then this is also indicated. The column "your earnings" displays the points you have earned. Recall that your match this period may be a different person than your match in the previous period. Please write the results on your record sheet under the appropriate headings. 
Figure C: Screen for the results of the period:

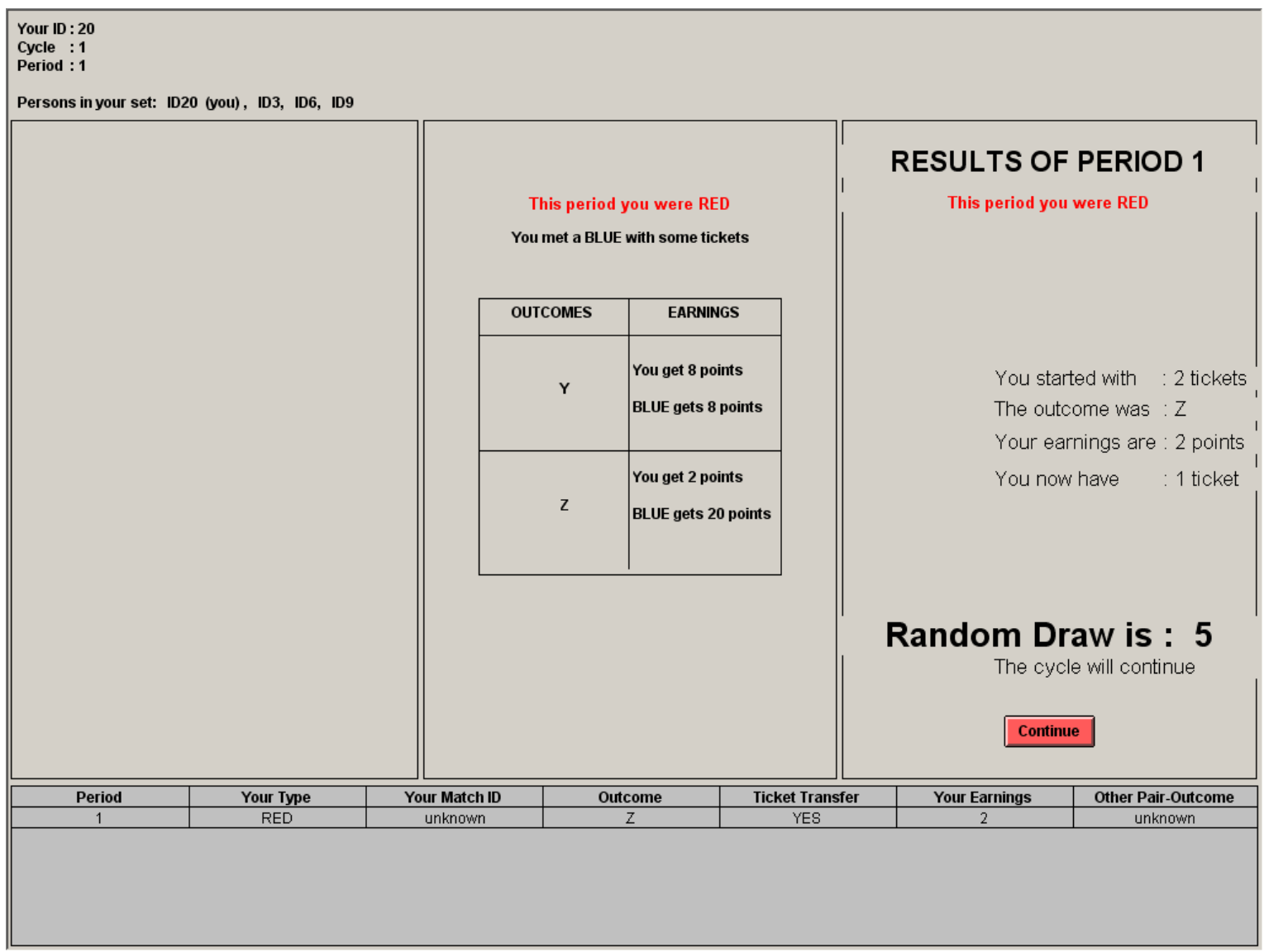

\section{Reminder on the duration of the experiment}

There will be five cycles of unknown duration. The duration of each cycle will be random. At the end of each period, the computer program will randomly select an integer number between 1 and 100, and show it on your screen (Figure C).

- If this random number is $1,2, \ldots$, or 93 , then the cycle will continue.

- If this random number is $94,95, \ldots$, or 100 , then the cycle will end.

Therefore, after any period there is always a $93 \%$ chance that the cycle will continue. This implies that, no matter what period you have reached, the expected number of additional periods is about 13. The number of past periods does not influence the chance that a cycle will end because the random procedure is exactly the same in every period.

When a cycle ends, you will be notified in a new screen. The rules in each cycle are the same but you interact with different persons in each cycle. After each cycle, new sets of persons will be formed. You will never interact with another participant for more than one cycle. 


\section{Final Comments}

- Do not talk to others and do not look at their screens.

- In every period you have a $50 \%$ chance to be red and $50 \%$ chance to be blue.

- If you are red, then you can choose to execute either outcome $\mathbf{Y}$ or $\mathbf{Z}$. If you are blue, then you may hold or transfer a ticket. Earned points will be redeemed for dollars. Tickets will not be redeemed for dollars.

- Your match is a random person in your set. You have one chance out of three of being matched with the same person in two consecutive periods.

- Independently of the period reached, there is a $93 \%$ chance of an additional period in the cycle, and a $7 \%$ chance that the cycle ends.

- The rules are the same in all five cycles. After a cycle, you will never interact with the same participants.

\section{Questions?}

Now is time for questions. Do you have any questions before we begin the experiment? 


\section{QUIZ}

15. The total number of cycles is

16. You are in period 1 of a cycle. What is the probability that the cycle will continue? How many additional periods do we expect?

17. What if you are in period 20? Probability Expected additional periods

18. The number of participants in the experiment (total in the room) is

19. In a given cycle, how many participants are in your set?

20. In each period how many participants do you interact with?

21. Will you ever see the ID of your match?

22. Can you see how many times your match chose $\mathrm{Y}$ or $\mathrm{Z}$ in the past? _No _If all pay _If I pay (circle one)

23. Will you know at the end of the period the outcome in the other pair from your set?

24. If IDs 5, $10 \& 16$ are in your set this cycle, is there any chance that ID 5, 10 or 16 will be your match in future cycles?

25. You are BLUE and your RED match executes $\mathbf{Y}$; how many points do you earn?

26. Suppose the experiment lasts 70 periods, you are RED half of the periods, BLUE half of the periods, and everybody always chooses $\mathbf{Y}$. How many dollars will you earn?

27. You are BLUE and you have a ticket. You choose "Give a ticket to red only if red executes Z." and your match chooses "Execute $\mathrm{Z}$ only if blue gives me a ticket." How many points do you and your match earn?

28. You are BLUE and you have a ticket., You choose "Keep your ticket(s)". Suppose your match chooses "Execute $\mathrm{Z}$ only if blue gives me a ticket." How many points do you and your match earn? 
29. Suppose the experiment lasts 70 periods, you are RED half of the periods, BLUE half of the periods, and everybody always chooses $\mathbf{Z}$. How many dollars will you earn?

ID

\begin{tabular}{|c|c|c|c|c|c|c|c|}
\hline Cycle & Period & $\begin{array}{l}\text { Your } \\
\text { Color }\end{array}$ & Tickets & $\begin{array}{c}\text { Your Choice } \\
\text { Blue: keep, give, } \\
\text { depends } \\
\text { Red: Y, Z, depends }\end{array}$ & $\begin{array}{c}\text { Outcome in } \\
\text { Your Pair } \\
\text { (Y or Z, ticket } \\
\text { transfer) }\end{array}$ & Earnings & $\begin{array}{l}\text { Cumulative } \\
\text { earnings }\end{array}$ \\
\hline & & & & & & & \\
\hline & & & & & & & \\
\hline & & & & & & & \\
\hline & & & & & & & \\
\hline & & & & & & & \\
\hline & & & & & & & \\
\hline & & & & & & & \\
\hline & & & & & & & \\
\hline & & & & & & & \\
\hline & & & & & & & \\
\hline & & & & & & & \\
\hline & & & & & & & \\
\hline & & & & & & & \\
\hline & & & & & & & \\
\hline & & & & & & & \\
\hline & & & & & & & \\
\hline & & & & & & & \\
\hline & & & & & & & \\
\hline & & & & & & & \\
\hline & & & & & & & \\
\hline & & & & & & & \\
\hline & & & & & & & \\
\hline & & & & & & & \\
\hline & & & & & & & \\
\hline & & & & & & & \\
\hline & & & & & & & \\
\hline & & & & & & & \\
\hline & & & & & & & \\
\hline & & & & & & & \\
\hline & & & & & & & \\
\hline & & & & & & & \\
\hline
\end{tabular}


Date

Experimental ID \#

\section{QUESTIONNAIRE}

This questionnaire is anonymous. The answers will help us in our research. Please answer them as accurately as possible. Your name will not be associated with the answers you are about to give.

1) Sex

$\mathrm{M} \square \mathrm{F}$

2) Major

3) Do you regularly use a credit card?

Yes

No

4) Consider the following hypothetical situation:

You have \$10. You can invest any portion of this amount and keep the rest. There is a 50\% chance that the investment pays $\$ 2.5$ per dollar invested, and a 50\% chance that it pays nothing.

How much would you invest?
Less than $\$ 2$
$\$ 2$ to $\$ 4$
$\$ 4$ to $\$ 6$
$\$ 6$ to $\$ 8$
more than $\$ 8$

5) Do you follow the financial news?

$\square \quad$ Yes

No

6) Were the instructions clear?

$\square \quad$ Yes

No

7) Please write here any comments/suggestions you may have about the experiment:

Thank you! 